\title{
Dark Matter search with Ge detectors
}

\author{
Paul L. Brink ${ }^{1}$ \\ Dept. of Physics, Stanford University \\ 382 Via Pueblo Mall, Stanford, CA 943405, USA \\ E-mail: pbrinkestanford.edu
}

The direct detection of Dark Matter, the relic abundance of weakly-interacting particles in the Universe responsible for large-scale galactic clustering, and inferred from studies of weaklensing and the cosmic microwave background, requires very sensitive detectors here on Earth. The Cryogenic Dark Matter Search (CDMS) collaboration has lead the field for most of the last decade in setting increasingly sensitive exclusion limits on the cross-section of these particles with ordinary baryonic matter.

The detectors used by CDMS are crystals of Ge, cooled to sub-Kelvin temperatures and instrumented with advanced phonon sensors to detect the small energy depositions, of order $10 \mathrm{keV}$, from these particles recoiling off the target nuclei. The expected event rate is less than 1 event per $\mathrm{kg}$ of target material per year, requiring significant measures to shield the detectors from radioactive backgrounds and powerful discrimination techniques. A description of the CDMS detector technology will be given, along with the present status of the field, and future plans for continuing the search for the Dark Matter of the Universe.

VERTEX 2009 (18 ${ }^{\text {th }}$ workshop) - VERTEX 2009

Veluwe, the Netherlands

September 13-18, 20

1 Speaker 


\section{Introduction}

The concordance model of Cosmology ${ }^{1}$ suggests that $23 \%$ of the mass-energy of the Universe is in the form of cold (non-relativistic) dark matter particles that only interact with ordinary matter via the nuclear-weak interaction. The relic density of these particles in our galactic neighbourhood is estimated to be $0.3 \mathrm{GeV} / \mathrm{cm}^{3}$ from the spiral arms' velocity rotation curve $^{2}$. Recent surveys ${ }^{3}$ confirm that these particles are in an approximate Maxwellian velocity distribution, with a circular velocity of $210 \mathrm{~km} / \mathrm{s}$ and a velocity cut-off of $\sim 550 \mathrm{~km} / \mathrm{s}$.

The detection of annihilation products from these dark matter particles constitutes the indirect searches (e.g. the GLAST/Fermi satellite ${ }^{4}$, VERITAS array ${ }^{5}$, and others are discussed in Ref. 6). Here we will focus on direct detection of Weakly Interacting Massive Particles (WIMPs) in the Ge crystals of the Cryogenic Dark Matter Search (CDMS) collaboration ${ }^{7}$. The interaction rate of these WIMPs with nucleons is expected to be less than 1 event per $\mathrm{kg}$ of target, per year ${ }^{2}$. Presently CDMS II sets the most stringent exclusion limit for the spinindependent (scalar) WIMP-nucleon cross-section for WIMPs above a mass of $42 \mathrm{GeV} / \mathrm{c}^{27}$.

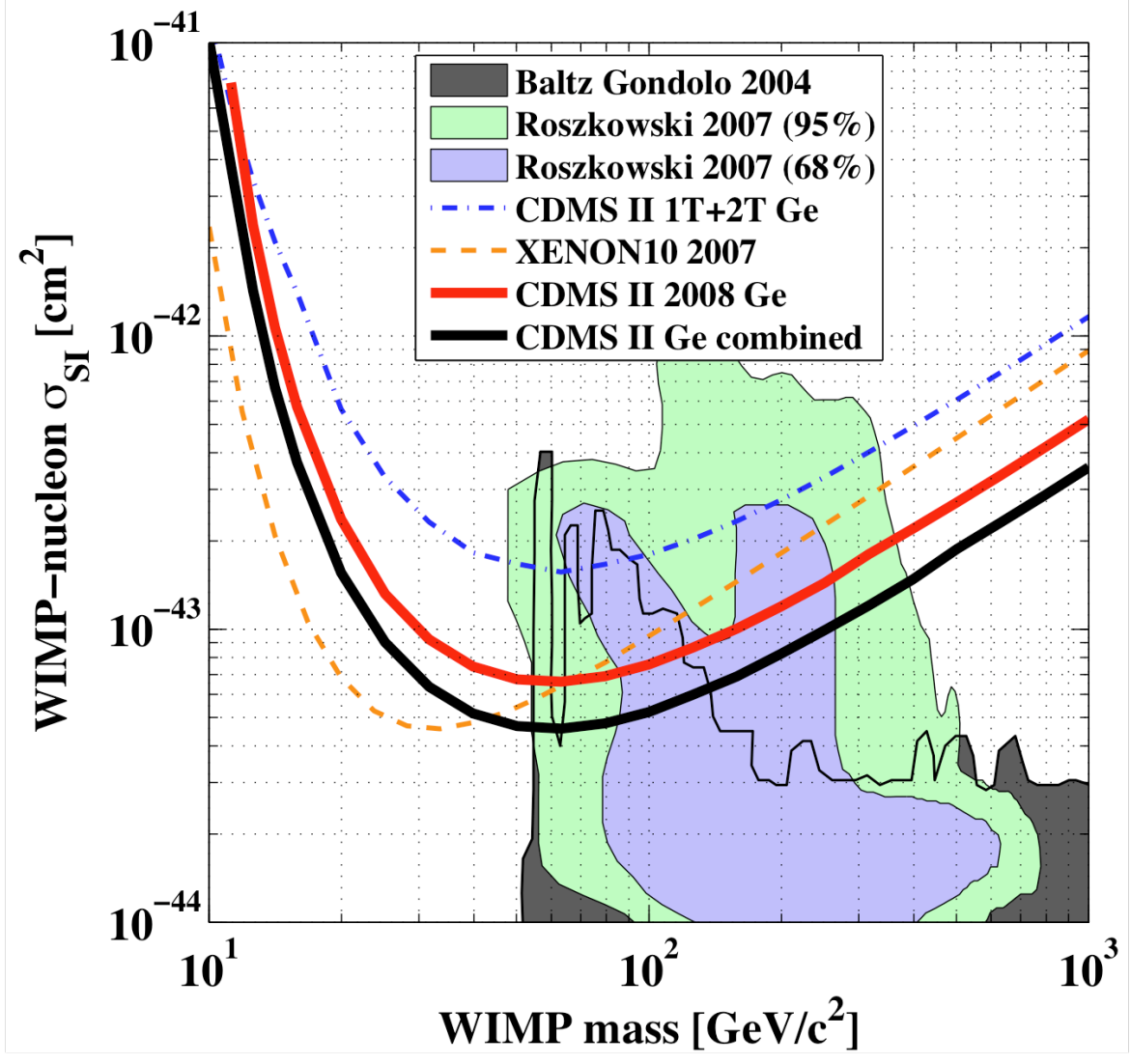

Figure 1: Present WIMP-nucleon scalar cross section upper limits (90\% CL) from the leading direct detection experiments. The shaded regions represent the favoured parameter space in supersymmetric extensions to the Standard Model (See Ref. 7). 


\section{Direct-detection Backgrounds}

A collection of recent direct-search exclusion limits are plotted in Fig. 1 (taken from Ref. 7) as a function of the WIMP mass for the case of spin-independent interaction. CDMS (and EDELWEISS $^{8}$ ) use Ge semiconductor crystals as the target, whereas liquid Xenon was used in the XENON $10^{9}$ and ZEPLIN III ${ }^{10}$ experiments. Most direct searches face the difficult challenge of distinguishing background events from the sought-after nuclear recoils due to WIMP particles scattering.

First, the experiments are housed at deep underground sites to reduce the incident flux of cosmic rays, in particular muons which can generate spallation products such as neutrons. The neutrons generated could have the correct energy to penetrate the shielding of the apparatus and recoil off target nuclei generating a signal very similar to that sought from WIMPs. Neutrons can also be generated from U/Th decay in the surrounding rock and materials via the $a-n$ reaction. CDMS-II was designed to operate in the Soudan Mine, Minnesota, at a depth of 2300 $\mathrm{ft}$, with an active plastic-scintillator veto shield and $40 \mathrm{~cm}$ of passive polyethylene shielding to moderate neutron backgrounds to a level far below the CDMS II WIMP-search goals: less than 1 single-scatter event per $35 \mathrm{~kg}$-years ${ }^{11}$ in the recoil energy range of interest (below $40 \mathrm{keV}$ ).

Background events due to electromagnetic interactions are orders of magnitude higher in rate, thus all these experiments have deployed techniques to discriminate between nuclear recoils (due to neutrons or WIMPs) vs electron recoils (due to X-rays, gammas, electrons). In the case of the noble-liquid experiments ${ }^{9,10}$ the relative light yields for the initial scintillation $v s$ that from detecting the ionization generated by the initial event allows one to distinguish between electron and nuclear recoils. For indirect-gap semiconductors like Ge, similar discrimination power can be achieved by measuring the event recoil energy and comparing to the ionization signal, as in $\mathrm{CDMS}^{7}$ and EDELWEISS ${ }^{8}$. For Ge at the recoil energies of interest a third of the energy is in the form of ionization for electron recoil events, whereas for nuclear recoils it is only a tenth. For CDMS the obtained rejection power is greater than $10^{5}$, and is adequate for a $25 \mathrm{~kg}$ Ge target operated for several years ${ }^{11}$.

Of more concern is an electromagnetic background due to electrons incident on the surface of the Ge crystals. Both the CDMS II and EDELWEISS-I detectors have a surface 'dead-layer' of $\sim 1$ micron within which electron-recoils events do not generate the full ionization signal expected, due to electron back-diffusion ${ }^{12}$. Note that the voltage applied across the Ge crystal for the ionization measurement needs to be kept small, a few Volts, otherwise the drifting electrons and holes would generate a large population of phonons that would swamp the discrimination technique of identifying bulk electron $v s$ nuclear recoil events by the ratio of ionization signal to intrinsic recoil phonon energy. Surface electron recoil events with their suppressed ionization signal can mimic the sought-after nuclear recoil signal. However for CDMS-II (and SuperCDMS) this limiting background has been overcome by using athermal phonon sensors, which not only measure the total recoil energy but are designed to measure the phonon signal before significant thermalization in the crystal has occurred. The phonon signal pulse shapes tend to be faster for events near the surface of the crystals (due to enhanced anharmonic phonon decay, with the resultant phonons at lower, but still detectable, frequency propagating through the crystal more quickly) allowing the rejection of such surface events with 
a discrimination ability of several hundred. These Z-dependent Ionization and Phonon (ZIP) detectors of CDMS II allowed the competitive results shown in Fig. 1 to be obtained where zero events were observed in the net exposure of $\sim 120 \mathrm{~kg}$-days.

\section{CDMS Detector Technology}

The target of CDMS-II consisted of 19 discs of Ge and 11 discs of Si (The Si allowed an independent measurement of any backgrounds) each $10 \mathrm{~mm}$ thick and $76 \mathrm{~mm}$ in diameter. One surface was instrumented with the athermal phonon sensors introduced above, and shown in Fig 2; the other surface was patterned into electrode grids to enable the bias for the ionization measurement. The grid was segmented to give a fiducial volume of $\sim 82 \%$ surrounded by an outer, guard-electrode, grid with its own charge-sensitive amplifier. Any events in this outer volume of the crystal were assumed to be incident radioactive background and were vetoed. The readout electronics and associated cryogenic hardware for CDMS II and the first future stages of the SuperCDMS program are described in detail in Ref. 13. The detectors themselves are fabricated with CMOS-style photolithography adapted for the thick substrates and the required double-sided photolithography ${ }^{14}$.

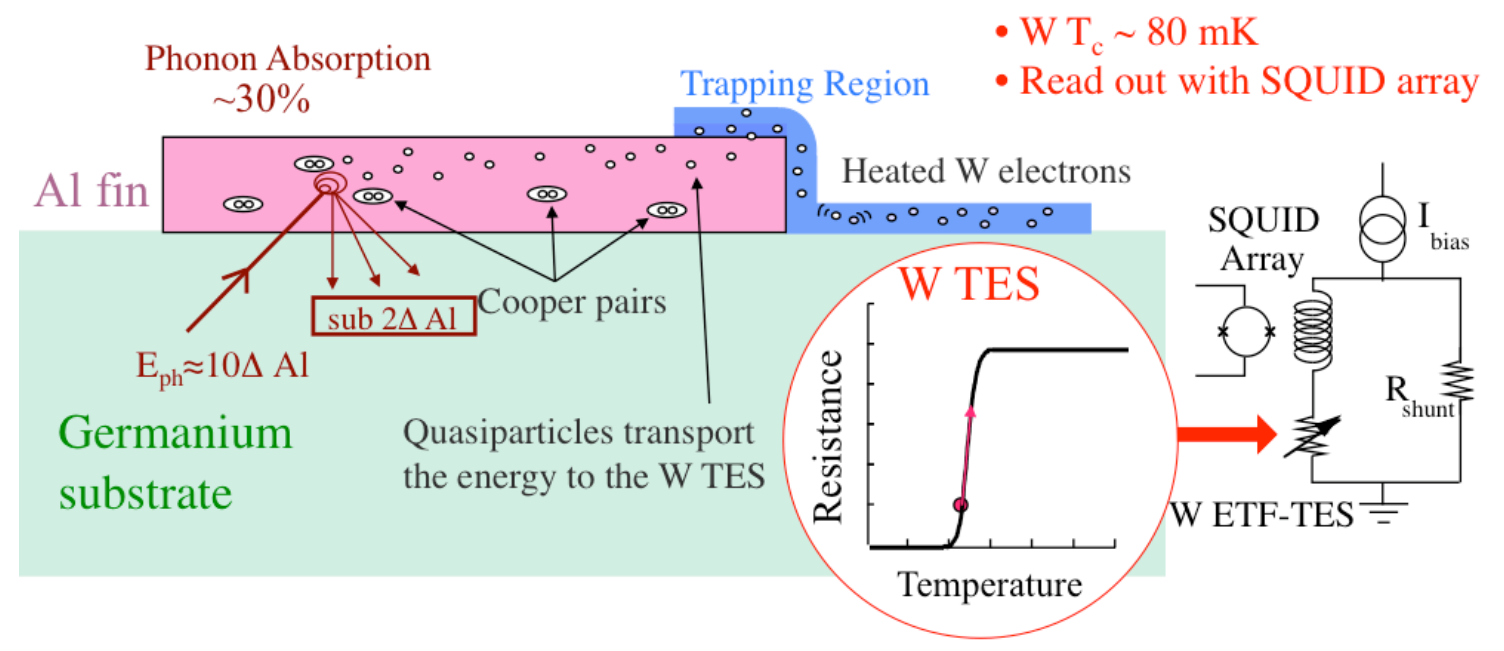

Figure 2: Schematic of the response of the CDMS-style athermal phonon sensors to incident phonon flux from events in the underlying Ge target. The flux arrives over a time scale of tens of microseconds with over $90 \%$ of the phonons having sufficient frequency $(>84 \mathrm{GHz}$ ) to break bound electron pairs (Cooper pairs with binding energy $2 \Delta$ ) in the superconducting Al films, which serve the function of being absorber fins. The liberated electrons (quasiparticles) then drift through the fins until they encounter the $\mathrm{W}$ traps. Here they inelastically scatter off a thermal population of electrons in the $\mathrm{W}$ (at a temperature of $\sim 80$ $\mathrm{mK}$ ). The $\mathrm{W}$ transition edge sensor (TES) is biased at its superconducting-normal transition temperature functioning as a very sensitive thermometer with a large change in resistance as the electron temperature changes. The change in resistance is measured with a Superconducting Quantum Interference Device (SQUID) Array, which is operating as a current-sensitive amplifier.

\section{SuperCDMS program}

The data-taking with the CDMS-II target has been completed. The analysis of this data is nearly complete with a submission of new WIMP-search results imminent at the time of writing. We have started upgrading the target at Soudan as part of the follow-on SuperCDMS program 
by replacing the detector 'towers' of CDMS-II with the new 'SuperTowers' of SuperCDMS, as depicted in Fig. 3.

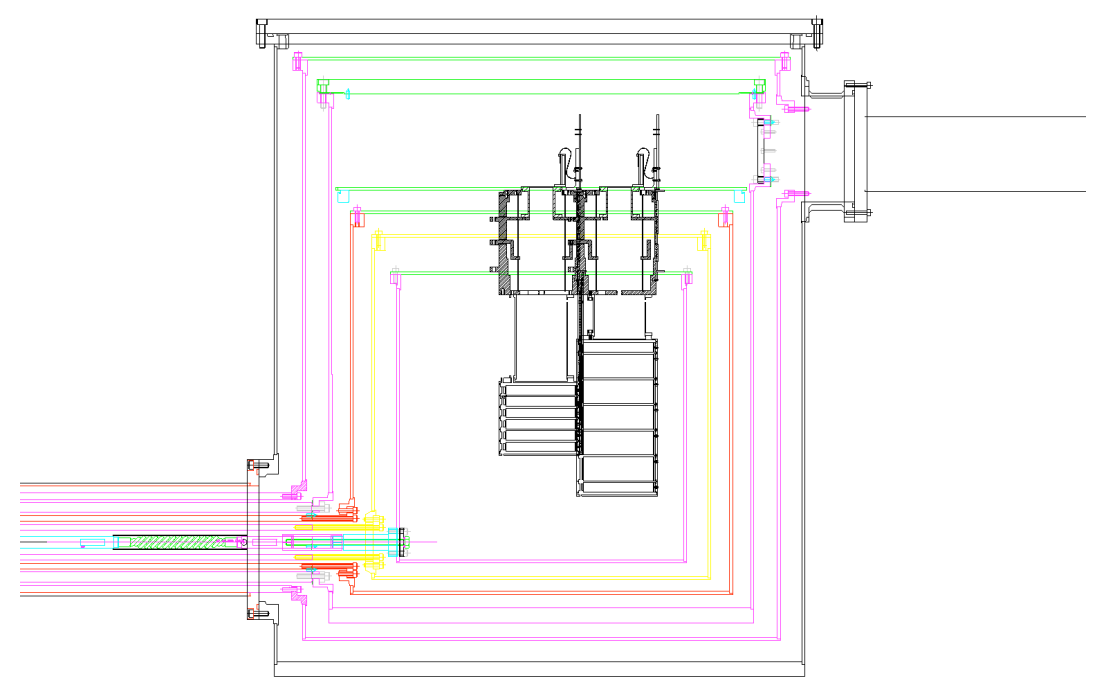

Figure 3: Cross-section of the CDMS-II Soudan ice-box showing a SuperTower (right) containing five 1inch thick Ge detectors, next to a CDMS II tower (left) that contains six $10 \mathrm{~mm}$ thick Ge detectors. The innermost can housing the detectors is at a temperature of $40 \mathrm{mK}$. The attachment of the icebox to the dilution refrigerator (not shown) is to the left and the detector electronics readout-stem is to the right. The top of tower bodies house the SQUID array amplifiers, and the FETs required for the ionization measurement.

The SuperCDMS towers contain improved Ge detectors ${ }^{12}$ with thicker substrates $(25 \mathrm{~mm})$ and improved phonon sensor designs that a cover larger effective surface area of the Ge. The aggregation of the phonon sensors into read-out channels has been re-arranged from the fourquadrants style of CDMS II into one where 3 inner channels are surrounded by an outer-ring comprising the fourth phonon sensor readout channel. This rearrangement gives enhanced radial information on the event location and improved identification of surface events ${ }^{12}$. The discrimination performance of these new detectors is expected to be adequate for the SuperCDMS Soudan zero-background event goal, with an expected sensitivity (90\% CL) of $5 \mathrm{x}$ $10^{-45} \mathrm{~cm}^{2}$ for the WIMP-nucleon scalar cross-section after operation for 3 years.

\section{Higher-performance Ge detectors for a Dark Matter Search}

Beyond 2012, SuperCDMS anticipates moving to a deeper site, for example SNOLab in Canada, and commissioning a cryostat and associated shielding for a $100 \mathrm{~kg}$ scale Ge target with expected reach of $3 \times 10^{-46} \mathrm{~cm}^{2}$. This sensitivity goal is similar to the expected sensitivity of tonne-scale noble-liquid target Dark Matter search experiments; and would allow a complementary search for Dark Matter using different target nuclei. Although a deeper site and more passive shielding satisfies the neutron backgrounds budget ${ }^{11}$ for future phases of SuperCDMS, further advances in Ge detector performance are required in order not to be limited by surface electron events.

For the last few years we have explored an alternative arrangement of the ionization electrodes suggested by P. Luke (see Ref. 15 and references therein) to allow both the athermal phonon sensors and the ionization measurement channels to identify surface events. Each face 
of the Ge disc is patterned with alternating bias and ground ionization electrodes. Events in the bulk of the crystal generate signals in charge-readout channels on opposing faces; whereas events near a surface generate a signal in only one charge-readout channel due to the tangential electric fields present.

We have recently demonstrated the performance of such a Ge detector ${ }^{15}$ where the detector is referred to as the iZIP concept. A photo of a packaged iZIP detector is shown in Fig. 4. Each detector has a mass of $0.6 \mathrm{~kg}$ with both outer charge electrodes and phonon channels able to veto events towards the perimeter, leaving a fiducial volume of $90 \%$.

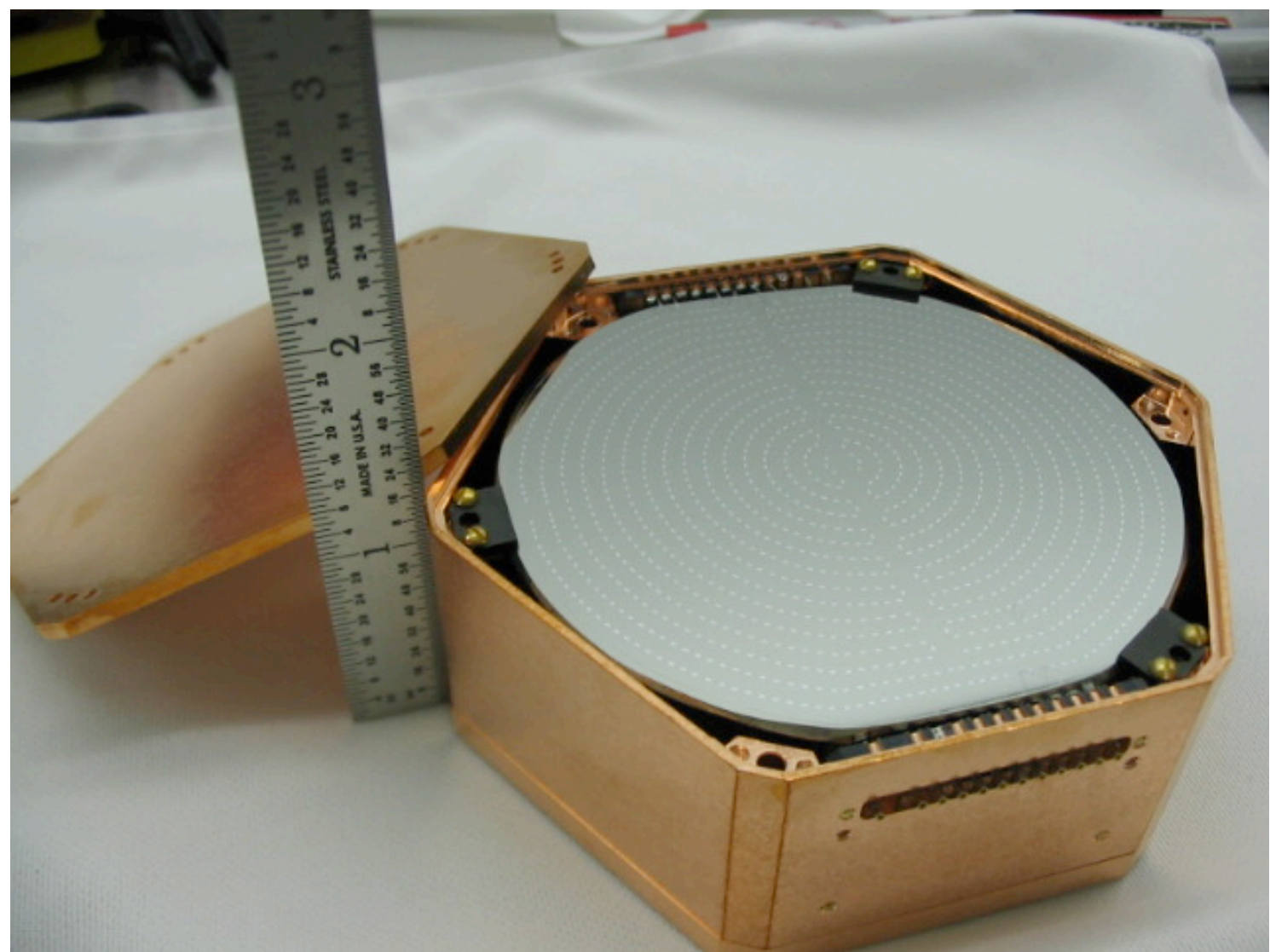

Figure 4: Prototype iZIP detector packaged in its hexagonal copper housing. The phonon sensors on the Ge surface are discernable as the lighter-shade arcs spaced $\sim 2 \mathrm{~mm}$ apart and arranged concentrically. Two phonon channels on the top surface allow determination of the $\mathrm{x}$-coordinate of the event. The two phonon channels on the bottom surface are rotated by 90 degrees to allow determination of the y-coordinate. The relative signal difference between the top and bottom surface phonon signals gives the z-coordinate of the event. The phonon sensors serve as the ground reference for the ionization measurements. The interdigitated bias lines spaced inbetween the phonon sensors for the ionization measurement are too narrow to be visible in this photograph.

The ratio of the top and bottom face ionization signals (referred to as the "charge symmetry' discrimination handle ${ }^{15}$ ) is alone responsible for a rejection power of $\sim 1000$ for surface electron events. We also appear to have gained a benefit from removing most of the amorphous Si film required to protect the Ge during fabrication, with the simple ionization yield performance (ratio of ionization to phonon signals for surface electron events) being superior 
for these iZIP detectors compared to those used for CDMS II or the EDELWEISS interleaved electrode demonstration ${ }^{8}$.

The true rejection capability of surface electron events by the iZIP detector ${ }^{15}$, (including the athermal phonon signal shapes and ionization yield) cannot be determined from testing above ground due to the ambient neutron background present. However the rejection power is expected to be $>3 \times 10^{5}$ for surface electron events based upon results from the EDELWEISS interleaved electrode detector demonstration at their underground site at the Modane Laboratory ${ }^{8}$.

\section{Conclusion}

The success to date of the CDMS II experiment in its search for Dark Matter has been driven by the use of advanced detector technology where the focus is on maximum information for each candidate recoil event. The burden of proof for a Dark Matter discovery is very high. Demonstration that candidate events are not natural backgrounds but instead are nuclear-recoil in nature, are single-scatter-site events, and are distributed in energy as anticipated, are all requirements. The use of different target nuclei materials is also desirable for verification.

The SuperCDMS collaboration has been approved to upgrade the CDMS II Ge target to 15 $\mathrm{kg}$ at the Soudan site with improved detectors and continue operations there for the next few years. The expected sensitivity of the search will bring us close to some of the most interesting parameter space of Supersymmetry anticipated by theorists shown in Fig. 1.

Going further, we plan to propose the iZIP detector concept for a $100 \mathrm{~kg}$ scale Ge target to be deployed at the SNOLab site. Further into the future a tonne-scale Ge detector, housed at the US Deep Underground Science and Engineering Laboratory (DUSEL), appears to be technologically feasible with R\&D funding already obtained by the GEODM collaboration. We are entering a fruitful era in the direct search for the Universe's Dark Matter.

\section{References}

[1] D.N. Spergel et al. (WMAP Collab.), Wilkinson Microwave Anisotropy Probe (WMAP) Three Year Results: Implications for Cosmology, Astrophys. J. Suppl. 170 (2007) 337.

[2] G. Steigman and M.S. Turner, Cosmological constraints on the properties of weakly interacting massive particles, Nucl. Phys. B 253 (1985) 375.

[3] L.E. Strigari and R. Trotta, Reconstructing WIMP Properties in Direct Detection Experiments Including Galactic Dark Matter Distribution Uncertainties, (2009) ArXiv : 0906.5361 v1.

[4] G. Dobler et al., The Fermi Haze: A Gamma-ray counterpart to the Microwave Haze, submitted to $A p J(2009)$ ArXiv: astro-ph.HE/0910.4583v1.

[5] R.G. Wagner et al. (VERITAS Collab.), Indirect Dark Matter Searches with VERITAS, to be published in proceedings of $31^{\text {st }}$ International Cosmic Ray Conference (ICRC), Lodz, Poland (July 2009) ArXiv: astro-ph.HE/0910.4563v1.

[6] W. de Boer, Indirect Dark Matter Searches in the Light of ATIC, FERMI, EGRET and PAMELA, invited talk at SUSY09, Boston, (2009) ArXiv: astro-ph.CO/0910.2601v1 and references therein. 
[7] Z.Ahmed et al. (CDMS Collab.), Search for Weakly Interacting Massive Particles with the First Five-Tower Data from the Cryogenic Dark Matter Search at the Soudan Underground Laboratory, Phys. Rev. Lett. 102, (2009) 011301.

[8] A. Broniatowski et al. (EDELWEISS Collab.), A new high-background-rejection dark matter Ge cryogenic detector, Physics Letters B 681, (2009) 305.

[9] E. Aprile et al. (XENON 10 Collab.), New measurement of the relative scintillation efficiency of xenon nuclear recoils below $10 \mathrm{keV}$, Phys. Rev. C 79, (2009) 045807.

[10] V. N. Lebedenko et al. (ZEPLIN III Collab.), Limits on the Spin-Dependent WIMP-Nucleon Cross Sections from the First Science Run of the ZEPLIN-III Experiment, Phys. Rev. Lett. 103, (2009) 151302 .

[11] D.S. Akerib et al. (SuperCDMS Collab.), Present Status of the SuperCDMS program, J. of Low Temp. Phys. 151, (2008) 818.

[12] C. N. Bailey et al. (SuperCDMS Collab.), Detector Development for the Next Phases of the Cryogenic Dark Matter Search: Results from 1 inch Ge and Si Detectors, J. of Low Temp. Phys. 151, (2008) 211; Z. Ahmed et al. (SuperCDMS Collab.), Characterization of SuperCDMS 1-inch Ge Detectors, to appear in AIP Conference proceedings for LTD13 Workshop, Stanford (July 2009).

[13] D.S. Akerib et al., Design and performance of a modular low-radioactivity readout system for cryogenic detectors in the CDMS experiment, Nucl. Instru. and Meth. A 591, (2008) 476; D.N. Seitz et al. (SuperCDMS), SuperCDMS Detector Readout Cryogenic Hardware, to appear in AIP Conference proceedings for LTD13 Workshop, Stanford (July 2009).

[14] P.L. Brink et al. (SuperCDMS Collab.), SuperCDMS Detector Fabrication Advances, to appear in AIP Conference proceedings for LTD13 Workshop, Stanford (July 2009).

[15] M. Pyle et al., Surface Electron Rejection from Ge Detector with Interleaved Charge and Phonon Channels, to appear in AIP Conference proceedings for LTD13 Workshop, Stanford (July 2009). 